\title{
Analgesic effect of non-pharmacological interventions in healthy newborns: A randomised controlled study
}

\author{
Guwani Liyanage $^{1}$, Dulani Gunasekara ${ }^{2}$, Ayomi Soodin $^{3}$, Manori Gamage ${ }^{1}$
}

Sri Lanka Journal of Child health, 2014; 43(3): 142-146

\begin{abstract}
Objectives: To assess if breastfeeding and skin-toskin contact have an analgesic effect in newborns undergoing venepuncture and to evaluate if skin-toskin contact together with breast feeding provides additional analgesic effect compared to skin-to-skin contact alone.
\end{abstract}

Method: We conducted a prospective randomised controlled study in three maternity units in Colombo South Teaching Hospital. Sixty infants who were undergoing routine venepuncture to assess serum bilirubin levels and who fulfilled the inclusion criteria were enrolled. They were randomly assigned to 1 of 3 study groups. In group I, infants were breastfed while being held in skinto-skin contact. In group II, babies were given skinto-skin contact by the mother. Control group infants (Group III) were kept on a table similar to the procedure table during venepuncture, which is the standard ward procedure. Two trained observers independently measured the pain score, using Premature Infant Pain Profile (PIPP).

Results: Pain scores of 20 neonates from each group were analyzed. There were no significant differences between the 3 groups in terms of demographic characteristics. Median PIPP scores of the 2 study groups were statistically significant when compared to the control group. There was no significant difference of pain score between the breast feeding with skin to skin contact and skin to skin contact groups.

Conclusions: Skin-to-skin contact and breast feeding were effective in reducing the pain during venepuncture. However, breastfeeding with skinto-skin contact did not provide additional analgesic effect compared to skin-to-skin contact alone.

${ }^{1}$ Senior Lecturer, ${ }^{2}$ Associate Professor in
Paediatrics, University of Sri Jayawardenapura,
${ }^{3}$ Consultant Paediatrician, Colombo South
Teaching Hospital

(Received on 3 September 2013: Accepted after revision on 25 October 2013)
(Key words: pain; newborn infant; skin to skin contact)

\section{Introduction}

Neonates have anatomical, physiological and hormonal components of nociception ${ }^{1}$. There is evidence to show that neonates do feel pain ${ }^{2}$. Pain during the neonatal period causes long term problems especially in preterm neonates ${ }^{3,4}$. Most importantly, prolonged pain can cause structural and functional changes in the central nervous system $^{5}$. Pain assessment tools are mostly based on behavioural changes alone and / or with physiological indicators such as heart rate and saturation ${ }^{6}$.

To alleviate pain, pharmacological methods such as topical anaesthetic creams or paracetamol have not been shown to be effective in neonates and have potential adverse effects when used $^{7,8}$. Non pharmacological interventions such as breast feeding $^{9}$ and skin to skin care ${ }^{10}$ are reported to be effective methods of analgesia. A randomized controlled trial has demonstrated that breast feeding during heel puncture gives lower PIPP scores than with sucrose $\mathrm{e}^{11}$.

\section{Objectives}

To assess whether breast feeding and skin to skin contact have an analgesic effect in newborns undergoing venepuncture and to evaluate whether breastfeeding together with skin-to-skin contact provided additional analgesia effect compared to skin-to-skin contact alone.

\section{Method}

This was a prospective, open randomised controlled study conducted in maternity units of Colombo South Teaching Hospital. Breastfed infants born at $\geq 35$ weeks of gestation with an Apgar score of seven or more at 5 minutes and who needed to undergo venepuncture to assess serum bilirubin levels were included. Babies with congenital anomalies, babies with severe illness 
requiring special care, babies who required more than single phototherapy for jaundice, babies with suspected sepsis with symptoms of lethargy, poor feeding and fever were excluded. Informed consent was obtained from the parents of eligible infants. The Ethics Committee of the Colombo South Teaching Hospital approved the study protocol.

Infants were randomly assigned to one of three study groups. Allocation into each group was done randomly with sealed envelopes. It was not possible to blind investigators and patients for the allocated group. In the two intervention groups (I \& II), infants were either breastfed while being held skin to skin (Group I) or given skin to skin contact without breast feeding (Group II). In the control group (Group III) infants were kept on a table similar to the procedure table in the maternity ward, which is used for cannulations and venepunctures.

Participating infants and their mothers were taken into a quiet room for venepuncture. Infants had a pulse oximeter probe fixed to the foot prior to the procedure. Infant's heart rate and oxygen saturation were monitored with an Ohmeda pulse oximeter. All 3 groups were monitored, starting 2 minutes before the venepuncture and continuing till the end of the procedure:

Group I: while breast feeding with skin to skin contact.

Group II: while being held in their mother's arms with skin to skin contact without breast feeding.

Group III: while infant was lying on the procedure table.

A nurse performed venepuncture on the dorsal aspect of the infant's hand with a 25 gauge butterfly needle. Two trained observers started recording the score for premature infant pain profile (PIPP) scale as soon as the needle was inserted.
Pain score

The PIPP scale ${ }^{12}$ is a multidimensional measure developed to assess acute pain in preterm and term infants. It measures gestational age, behavioural state, heart rate, oxygen saturation and 3 facial reactions (brow bulge, eye squeeze, nasolabial furrow). These facial actions that have been observed in $99 \%$ of neonates within six seconds of painful procedures are believed to be the most sensitive indicators of infant pain ${ }^{13}$. In term infants, scores range from 0 (no pain) to 18 (maximum pain).

\section{Data collection}

A minimum 16 subjects were required for each group to detect a mean difference of four in the PIPP score in treatment groups compared to control group (CI- 95\%) of confident interval with a power of $80 \%$ for an equal variance of 16 . Demographic characteristics, including gestational age, birth weight, postnatal age, gender, type of delivery were collected on a standardized data collection form.

\section{Statistical Analysis}

Statistical analysis was performed with SPSS (Version 18). We used Kruskal-Wallis one way analysis of variance on scores to compare overall differences among three groups. Comparisons of median pain scores were performed using MannWhitney U tests. Because three pair wise planned comparisons were made we considered $\mathrm{P}<0.05$ as significant.

\section{Results}

Sixty-four neonates were eligible. Four were excluded since venepuncture failed at the first attempt. Twenty neonates were allocated to each group. Demographic characteristics were compared between groups by using Anova and Kruskal Wallis for continuous and categorical data. There were no significant differences between the 3 groups in terms of following characteristics (Tables $1 \& 2)$

Table 1: Demographic characteristics of the study subjects

\begin{tabular}{|l|c|c|c|c|}
\hline \multicolumn{1}{|c|}{ Factor } & Control & Group A & Group B & P value \\
\hline $\begin{array}{l}\text { Gestational age in days } \\
\text { at birth (IQR) }\end{array}$ & $\begin{array}{c}271 \\
(262-279)\end{array}$ & $\begin{array}{c}262 \\
(256-280)\end{array}$ & $\begin{array}{c}270 \\
(259-279)\end{array}$ & 0.919 \\
\hline Birth weight* (SD) & 2.7135 & 2.97975 & 2.58425 \\
$(0.529)$ & $(0.487)$ & $(0.557)$ & 0.0607 \\
\hline Age at the time of the & $18(22)$ & $33(31)$ & $32(41)$ & 0.235 \\
\hline
\end{tabular}

*ANOVA 
Table 2

Nominal characteristics of the study subjects

\begin{tabular}{|c|c|}
\hline Factor & P value \\
\hline Mode of delivery & 0.495 \\
\hline Sex & 0.936 \\
\hline
\end{tabular}

Median PIPP scores of the breast feeding with skin to skin contact group (Treatment A in Figure 1), skin to skin contact only group (Treatment $\mathrm{B}$ in Figure 1), the control group and their interquartile ranges are shown in Figure 1. The levels of significance in comparison of the 3 groups are shown in Table 3.

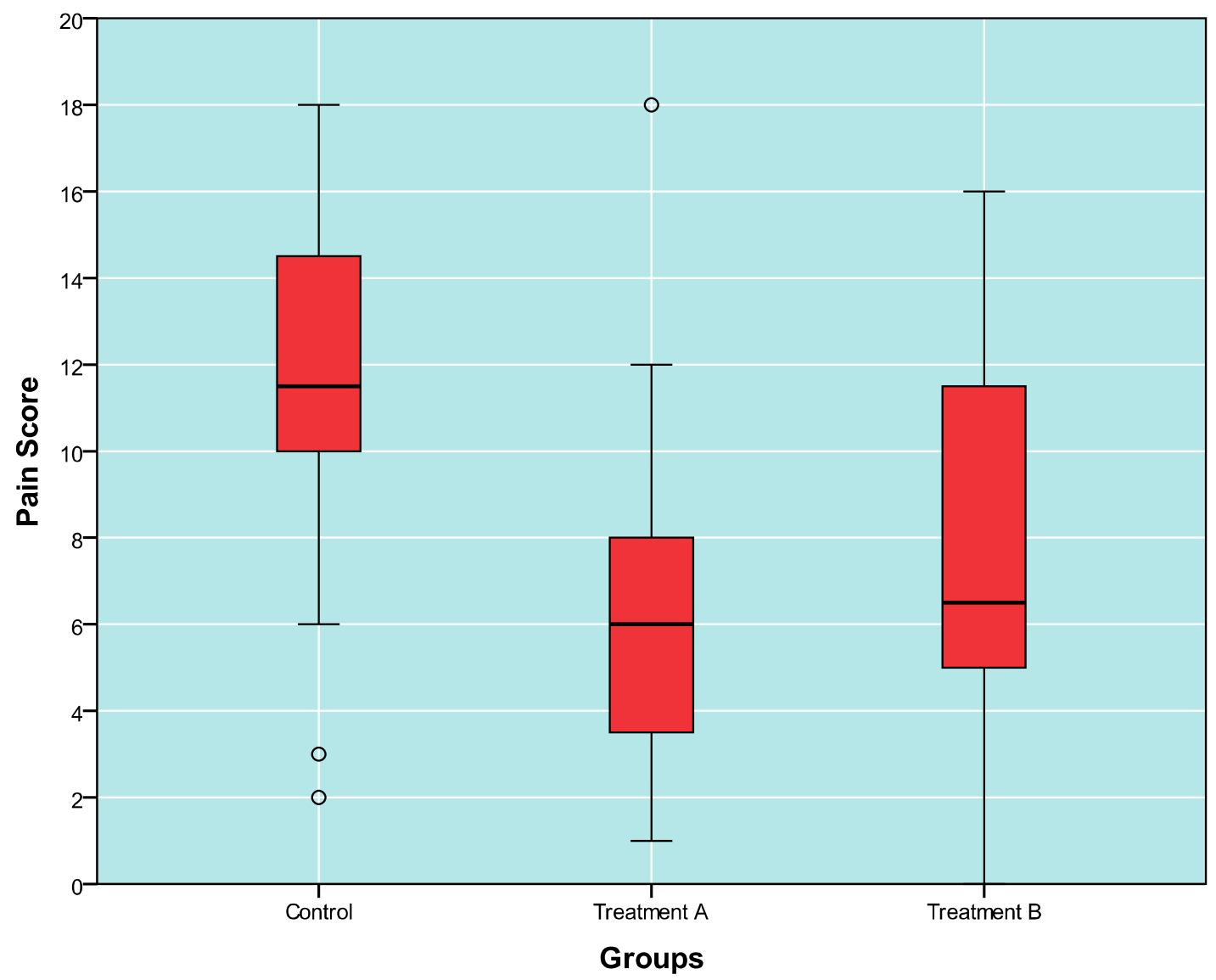

Figure 1: Pain during venepuncture in 60 infants according to PIPP scale (0 to 18) showing median values and interquartile ranges

(Treatment A: breast feeding with skin to skin contact group. Treatment B: skin to skin contact only group)

Table 3: Comparison of the 3 groups and the levels of significance

\begin{tabular}{|l|c|}
\hline \multicolumn{1}{|c|}{ Comparison } & P value \\
\hline Control group and breast feeding only group & $<0.001$ \\
\hline Control group and skin to skin contact with breast feeding group & 0.012 \\
\hline Breast feeding only and skin to skin contact with breast feeding group & 0.271 \\
\hline
\end{tabular}

\section{Discussion}

$5 \%$ lignocaine ointment, lidocaine-prilocaine cream $^{7}$ (EMLA) cream and paracetamol ${ }^{8}$ have not demonstrated a decrease in pain response to heel prick. There is substantial literature on the effective analgesia of skin-to-skin ${ }^{9}$ contact, breastfeeding ${ }^{10}$, sweet taste ${ }^{11}$ and milk flavor in reducing procedural pain in neonates. A recent meta-analysis showed that sucrose is safe and effective for reducing procedural pain from single events and an optimal 
dose could not be identified due to inconsistency in effective sucrose dosage among studies ${ }^{14}$.

Gray et $\mathrm{al}^{9}$ reported that breast feeding before, during, and after heel prick markedly reduced crying and grimacing and prevented the increase in heart rate in term neonates compared with swaddled infants in their cots. Recent Cochrane systematic review has evaluated the effectiveness of breastfeeding or supplemental breast milk in reducing procedural pain in neonates and concluded that if available, breastfeeding or breast milk should be used to alleviate procedural pain in neonates undergoing a single painful procedure rather than placebo, positioning or no intervention. Administration of glucose/sucrose had similar effectiveness as breastfeeding for reducing pain ${ }^{15}$.

A randomized controlled trial has analyzed the effectiveness of skin-to-skin contact in decreasing pain from heel-lancing in healthy term neonates. It has also concluded that breast feeding with skin-toskin contact is more effective than skin-to-skin contact alone in reducing procedural pain ${ }^{16}$.

\section{Conclusions}

- Breast-feeding and skin to skin care during painful procedures have an analgesic effect.

- Breast feeding with skin to skin contact, did not show an additional effect on relieving pain when compared to skin to skin contact without breast feeding.

We recommend that health care personnel involved in newborn care should be routinely trained to practise this simple intervention during basic invasive procedures such as venepuncture.

\section{References}

1. Anand K J, Hickey P R. Pain and its effects in human neonate and fetus. New England Journal of Medicine 1987; 319(21):132-1329.

2. Anand K J S. The International EvidenceBased Group for Neonatal Pain Consensus statement for the prevention and management of pain in the newborn Archives of Pediatrics and Adolescent Medicine 2001;155:173-80. http://dx.doi.org/10.1001/archpedi.155.2.173

3. Grunau RE, Holsti L, Peters J W. Long-term consequences of pain in human neonates. Seminars in Fetal and Neonatal Medicine 2006; 11(4):268-75. http://dx.doi.org/10.1016/j.siny.2006.02.007
4. Anand KJS. Clinical importance of pain and stress in preterm newborn infants. Biology of the Neonate 1998; 73:1-9. http://dx.doi.org/10.1159/000013953

5. Fitzgerald M, Beggs S. The neurobiology of pain: developmental aspects. Neuroscientist 2001; 7(3):246-57. http://dx.doi.org/10.1177/10738584010070030 $\underline{9}$

6. Mathew P J, Mathew J L. Assessment and management of pain in infants. Postgraduate Medical Journal 2003; 79(934):438-43. http://dx.doi.org/10.1136/pmj.79.934.438

7. Taddio A, Ohlsson A, Einarson T R, Stevens B, Koren G. A systematic review of lidocaineprilocaine cream (EMLA) in the treatment of acute pain in neonates. Pediatrics. 1998; 101(2):E1.

http://dx.doi.org/10.1542/peds.101.2.e1

8. Shah A, Taddio A, Ohlsson A. Randomised controlled trial of paracetamol of heel prick pain in neonates. Archives of Disease in Childhood Fetal and Neonatal Edition 1998; 79:F209-F211.

http://dx.doi.org/10.1136/fn.79.3.F209

9. Gray L, Watt L, Blass EM. Skin-to-skin contact is analgesic in healthy newborns. Pediatrics 2000; 105(1):E14.

http://dx.doi.org/10.1542/peds.105.1.e14

10. Analgesic effect of breast feeding in term neonates: randomised controlled trial British Medical Journal 2003; 326(7379): 13-16. http://dx.doi.org/10.1136/bmj.326.7379.13

11. Blass EM, Watt L. Suckling and sucroseinduced analgesia in human newborns. Pain 1999; 83(3):611-23. http://dx.doi.org/10.1016/S03043959(99)00166-9

12. Stevens B, Johnston C, Petryshen P, Taddio A. Premature infant pain profile: Development and initial validation. Clinical Journal of Pain 1996; 12:13-22. http://dx.doi.org/10.1097/00002508199603000 $-00004$

13. Ricardo CL, SoocramanienVeerapen, Sophie Couderc, MyriamJugie et al. Pain expression in neonates: facial action and cry. Pain 1987 ; 28:395-410. http://dx.doi.org/10.1016/03043959(87)90073$\underline{X}$ 
14. Stevens B, Yamada J, Ohlsson A. Sucrose for analgesia in newborn infants undergoing painful procedures Cochrane Database of Systematic Reviews 2010, Issue 1

15. Shah PS, Herbozo C, Aliwalas LL, Shah. Breastfeeding or breast milk for procedural pain in neonates Cochrane Database of Systematic Reviews 2012 Dec 12 http://dx.doi.org/10.1002/14651858.CD004950 .pub3
16. Okan F, Ozdil A, Bulbul A, Yapici Z, Nuhoglu A. Analgesic effects of skin-to-skin contact and breastfeeding in procedural pain in healthy term neonates. Annals of Tropical Paediatrics 2010; 30(2):119-28

http://dx.doi.org/10.1179/146532810X127039 $\underline{02516121}$ 